\title{
A Novel Missense Mutation in Human Receptor Roundabout-1 (ROB01) Gene Associated with Pituitary Stalk Interruption Syndrome
}

\author{
(D) Ziqin Liu, (D) Xiaobo Chen \\ Capital Institute of Pediatrics, Clinic of Endocrinology, Beijing, China
}

\begin{abstract}
What is already known on this topic?
Pituitary stalk interruption syndrome (PSIS) is a rare, congenital anomaly of the pituitary gland characterized by pituitary gland insufficiency, thin or discontinuous pituitary stalk, anterior pituitary hypoplasia, and ectopic positioning of the posterior pituitary gland. The underlying genetic etiology for the vast majority of cases remains to be determined.
\end{abstract}

\section{What this study adds?}

Heterozygous mutations of the human Receptor Roundabout-1 (ROBO1) gene were recently shown to be responsible for PSIS. Here, we used an exome sequencing approach to reveal a novel missense mutation (c.1690C > T, p.Pro564Ser) in ROBO1 gene in a 4 year boy with with PSIS, The mutation was carried by his mother, whose pituitary magnetic resonance imaging showed also an abnormality.

\begin{abstract}
Pituitary stalk interruption syndrome (PSIS) is characterized by the association of an absent or thin pituitary stalk, an absent or hypoplastic anterior pituitary lobe and an ectopic posterior pituitary (EPP) lobe. The causes of this anatomical defect include both genetic and environmental factors. Molecular genetic defects have been indentified in a small number of patients with PSIS. A 4-year-old boy presented with hypoglycemia and hyponatremia associated with growth hormone, thyroid stimulating hormone, and adrenocorticotropic hormone deficiencies. The patient had right sided strabismus. magnetic resonance imaging images showed pituitary hypoplasia, EPP and absent pituitary stalk. A novel Receptor Roundabout-1 (ROBO1) missense mutation (c.1690C > T, p.Pro564Ser) that may contribute to the disorder was found in this patient and his mother, who also exhibited pituitary abnormalities.

Keywords: Receptor Roundabout-1 gene, pituitary stalk interruption syndrome, combined pituitary hormone deficiency, missense mutation
\end{abstract}

\section{Introduction}

Pituitary stalk interruption syndrome (PSIS) is a rare disorder due to the blocked transportation of hormones from the hypothalamus to the pituitary. The estimated incidence of this disorder is $0.5 / 100,000$ births $(1,2)$. Patients with PSIS are characterized by a combination of specific pituitary hormone deficiencies. There are typical findings evident on cranial magnetic resonance imaging (MRI), including interrupted or thin pituitary stalk, absent or ectopic posterior pituitary, and anterior pituitary hypoplasia $(3,4)$. The diagnosis of this syndrome mostly depends on MRI imaging in combination with classical clinical and laboratory findings.
Despite intensive research into PSIS, the etiology remains unknown in $95 \%$ of cases, although genetic causes are suspected. In the human, mutations in LHX4, OTX2, HESX1, SOX3, PROKR2, GPR161 and CDON have been postulated to be associated with PSIS. Recently, mutations in the Receptor Roundabout-1 (ROBO1) gene have been reported in five patients with PSIS (5), confirming its genetic association with PSIS.

Here, we report a case of PSIS with multiple anterior pituitary deficiencies and the classical triad of MRI findings, in which whole exome sequencing (WES) analysis identified a novel heterozygous mutation in the $R O B O 1$ gene. This mutation is also carried by his mother, who also had abnormal pituitary 
function and short stature, thereby providing strong circumstantial evidence for the association between this variant and the familial pituitary abnormalities.

\section{Case Report}

The patient was a 4-year-old boy who was admitted to our hospital with an episode of generalized tonic-clonic seizures. The episode was not associated with fever or any sign of infection. He was born fullterm at 39 weeks gestation and delivered with normal birth parameters (weight 2860 g: 10th $25^{\text {th }}$ centile, length $50 \mathrm{~cm}$ : 50th centile). Psychomotor development was normal. At presentation the height of the patient was $97 \mathrm{~cm}$ ( < 3th centile) and his weight was 16 kg (10th-25th centile). Findings on physical examination were unremarkable apart from right strabismus (Figure 1). The penis was $2 \mathrm{~cm}$ (stretched) and the testicular volume was $1 \mathrm{~mL}$ bilaterally. The patient was the first child of nonconsanguineous Chinese parents. The father was healthy and of normal stature $\left(170.5 \mathrm{~cm}, 25^{\text {th- } 50 \text { th }}\right.$ centile), while the mother had a short stature $(146 \mathrm{~cm},<3$ th centile). No further details of the mother's medical history were available. His maternal grandmother also reported short stature and strabismus.

The patient was found to have severe hypoglycemia with a blood glucose concentration of $0.92 \mathrm{mmol} / \mathrm{L}$ (normal, 3.35.5) and severe hyponatremia with a blood sodium ( $\mathrm{Na}$ ) of $117 \mathrm{mmol} / \mathrm{L}$, (normal, 135-155). Hormone concentrations and biochemical parameters measured during hypoglycemia were as follows: serum insulin $0.9 \mathrm{mU} / \mathrm{L}$, serum cortisol 0.55 ug/dL (normal, 6.2-19.41), adrenocorticotropic hormone (ACTH) $9.8 \mathrm{pg} / \mathrm{mL}$, urine ketone bodies were negative, plasma lactate $1.8 \mathrm{mmol} / \mathrm{L}$ (normal, <2) and serum ammonia $52.2 \mathrm{~mol} / \mathrm{L}$ (normal, < 80). The levels of creatinine kinase, creatine kinase MB fraction, organic acids, amino acids, acylcarnitines and free carnitine in plasma were normal. Blood gas analysis was pH 7.44, $\mathrm{PCO}_{2} 29.6$ mmHg, $\mathrm{HCO}_{3} 22.4 \mathrm{mmol} / \mathrm{L}, \mathrm{BE}-0.6 \mathrm{mmol} / \mathrm{L}$. No abnormalities were detected on complete blood count. Hemoglobin A1C was $5.6 \%$ (normal, 4-6\%). Growth hormone (GH) deficiency was attributed to this patient after insulin tolerance test and L-dopa test, with a peak GH of 0.3 and $0.05 \mathrm{ng} / \mathrm{mL}$, respectively. Insulin like growth factor-1 (IGF-1) was 25 ng/ $\mathrm{mL}$ (normal, 66-427) while prolactin was in the normal

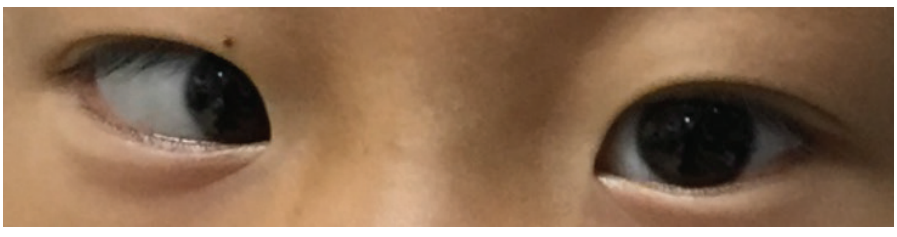

Figure 1. Image of the patient showing right strabismus range. In addition to $\mathrm{GH}$ deficiency, he was diagnosed with central hypothyroidism [free T4, 8.6 pmol/L (normal, 10.820), thyroid stimulating hormone (TSH): $0.489 \mathrm{ulU} / \mathrm{mL}$ (normal, 0.8-5)]. Luteinizing hormone (LH) was $0.13 \mathrm{IU} / \mathrm{L}$ and follicle-stimulate hormone (FSH) 0.7 IU/L. The karyotype was 46,XY. Echocardiogram, electroencephalogram (EEG) and video-EEG showed no abnormalities. Cranial MRI revealed a small anterior pituitary gland, absent pituitary stalk and an ectopic posterior lobe (Figure 2). A diagnosis of PSIS was made based on these clinical and laboratory findings. The patient presented with combined pituitary
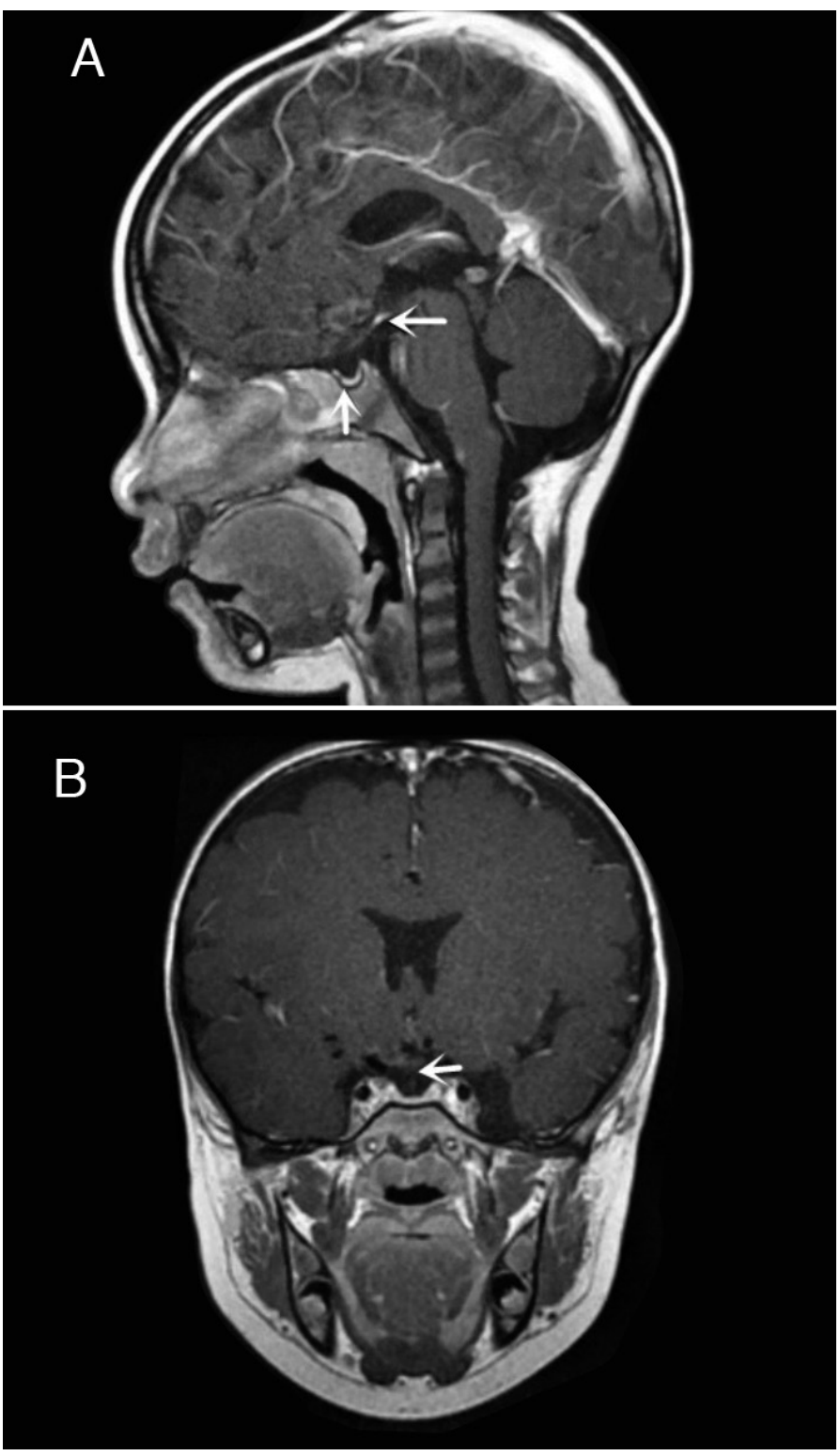

Figure 2. Sagittal and coronal magnetic resonance imaging of the pituitary confirming pituitary stalk interruption syndrome

(A) Sagittal view. The small anterior pituitary (vertical arrow) and the posterior lobe was localized at the hypothalamic region (horizontal arrow). (B) Coronal view. The pituitary stalk is absent 
hormone deficiency (CPHD) including GH deficiency, central hypothyroidism and central adrenocortical insufficiency. He was then treated with saline and hydrocortisone and a good response to this was obtained with stabilized blood sugar and blood Na concentrations. Subsequently, thyroxine (LT4) and $\mathrm{GH}$ replacement therapy were started.

\section{Genetic Analysis}

DNA samples obtained from the family were sequenced to identify the causal gene using WES. DNA was isolated from peripheral blood using DNA Isolation Kit (Bioteke Corporation, AU1802, Wuxi, China). Genomic DNA samples $(1 \mathrm{\mu g})$ were fragmented into 200-300 bp portions using a Covaris Acoustic System (Covaris, Woburn, MA, USA). The DNA fragments were then processed by end-repairing, A-tailing, adaptor ligation and a four-cycle pre-capture polymerase chain reaction amplification, after which all exons and the $50 \mathrm{bp}$ bases in their adjacent introns were captured by SeqCap EZ Med Exome Enrichment Kit (Roche, Madison, WI, USA). Post-capture amplification and purification was performed on the DNA library and then sequenced on an Illumina HiSeq X Ten platform (Illumina, San Diego, CA, USA) mannually. The raw data produced were then filtered and aligned with the human genome reference (hg19) using the BWA Aligner (http://bio-bwa.sourceforge. net/) and variants were identified using NextGene V2.3.4 software (Soft genetics, LLC, State College PA, USA). The data had a $151.24 \times$ mean read depth and about $97.95 \%$ of the targetbases were covered at $20 \times$ average read depth.
The filtered variants were then annotated by using NextGene V2.3.4 and the laboratory's own scripts to get related information, including the conservation of nucleotide bases and amino acids, prediction of the biological functions, frequency in normal populations (compared with 1000 Genomes, ExAC, dbSNP database and local specific databases), and the data from HGMD, Clinvar and OMIM. The potential effect of the variants were predicted by SIFT and Polyphen-2 $(6,7,8)$. All variants of pathogenicity were interpreted according to the American College of Medical Genetics standards and categorized (9). The ROBO1 gene has three transcripts recorded in the National Center for Biotechnology Information, of which NM_002941.3 was used as the reference sequence. Potentially pathogenic variants were verified using Sanger sequencing.

WES data filtering identified a heterozygous c.1690C > T, p.Pro564Ser variant (RefSeq: NM_002941.3; Chr3: 78717393) in the ROBO1 gene (Figure 3). Segregation studies revealed that the mother was also a carrier of the same mutation. This rare sequence variant was further predicted to be "probably damaging" with a score of 0.999 in Polyphen-2 and "damaging" with a score of 0.01 by SIFT. Multiple amino acid sequence alignments showed that p.Pro 564 is highly conserved in human, Chimps (Pan troglodytes), mice (Mus musculus), zebra fish (Danio rerio), frogs (Xenopus tropicalis) and chickens (G gallus) (Table 1).

The mother, who has short stature, also carried the same ROBO1 variant. She then underwent endocrine evaluation which showed an ACTH of $38.8 \mathrm{pg} / \mathrm{mL}$, cortisol of $8.1 \mathrm{ug} / \mathrm{dL}$,

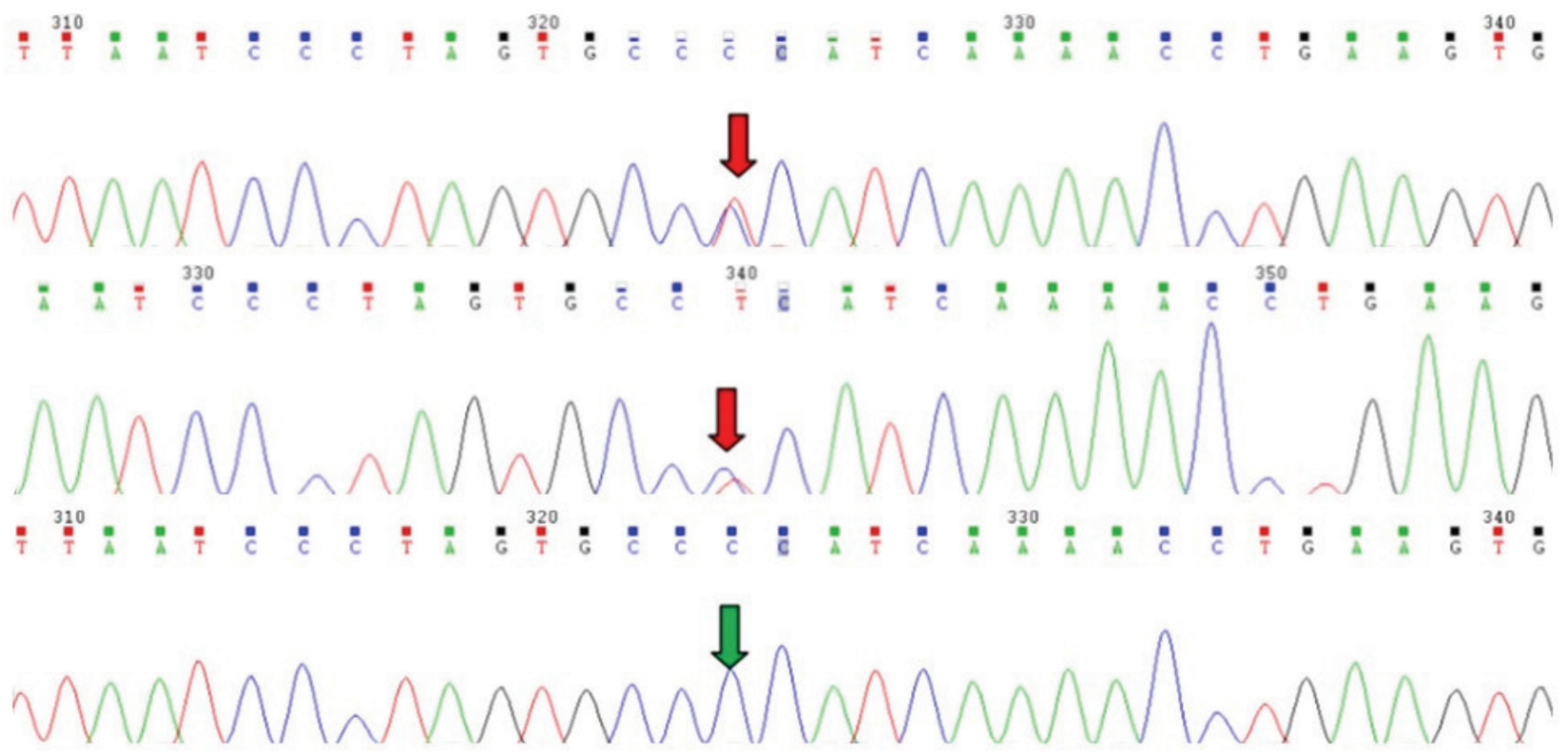

Figure 3. Sanger sequencing results of the family

The heterozygous c.1690C > T, Pro564Ser ROBO1 mutation is found in the patient (top) and his mother (middle). The father (bottom) has no mutation. The red arrows show the mutation 
IGF-1 of 188 ng/mL (115-307 ng/mL), FT4 9.4 pmol/L, TSH: $2.16 \mathrm{ulU} / \mathrm{mL}, \mathrm{LH}: 2.97 \mathrm{IU} / \mathrm{L}, \mathrm{FSH}: 5.34 \mathrm{IU} / \mathrm{L}$ and E2 of 67 $\mathrm{pg} / \mathrm{mL}$. Her pituitary MRI showed a thin pituitary stalk with hypoplasia of the adenohypophysis (Figure 4).

\section{Discussion}

The patient reported here had right strabismus and CPHD in $\mathrm{GH}, \mathrm{ACTH}$ and TSH. His mother, who also carried the variant, had abnormal pituitary function, short stature but normal eye structure while the patient's maternal grandmother had short stature and strabismus. Though DNA was not available from the deceased grandmother, this may reflect phenotypic variability in this family. The phenotypic variability found in the patient and his mother could be due to the impact of other genes in pituitary development or gene-environment interactions (10), and is similar reports of the missense variants involving HESX 1 and LHX4 genes (11), of which the heterozygous variants are characterized by highly variable phenotypes amongst family members.

To date, several etiological factors have been proposed for PSIS, and there is good evidence for a polygenic

\begin{tabular}{ll}
\hline $\begin{array}{l}\text { Table 1. Alignment of amino acid sequences encoded by } \\
\text { the ROBO1 gene from different species }\end{array}$ \\
\hline Species & Aa alignment \\
\hline Human & RPTDPNLIPSAPSKPEVTDVSRNT \\
P troglodytes & RPTDPNLIPSAPSKPEVTDVSRN \\
M musculus & RPTDPNLIPSAPSKPEVTDVSKN \\
D rerio & RPTDPNLIPSAPSKPDVTDVSRT \\
X tropicalis & RPTDPNLIPSAPSKPE \\
G gallus & RPTDPNLIPSAPSKPEVTDVSRN \\
\hline \multicolumn{2}{l}{ The P residue is highlighted in bold for each sequence } \\
\hline
\end{tabular}

etiology. HESX1, LHX4, OTX2, SOX3, and PROKR2 have been reported to be associated with PSIS $(12,13,14)$. In 2017, five unexplained PSIS cases including two famial cases identified one nonsense, one missense and one frameshift mutation (all heterozygous) in the ROBO1 gene by WES (5) and this report was the first to identify novel heterozygous frameshift, nonsense and missense variants (p.Ala977Glnfs*40, two affected sibs; p.Tyr 1114 Ter, sporadic case and p.Cys240Ser affected child and paternal aunt) in ROBO1 gene (Table 2) (15). Of these five cases, three showed isolated GH deficiency and the other two presented with combined GH and TSH deficiencies.

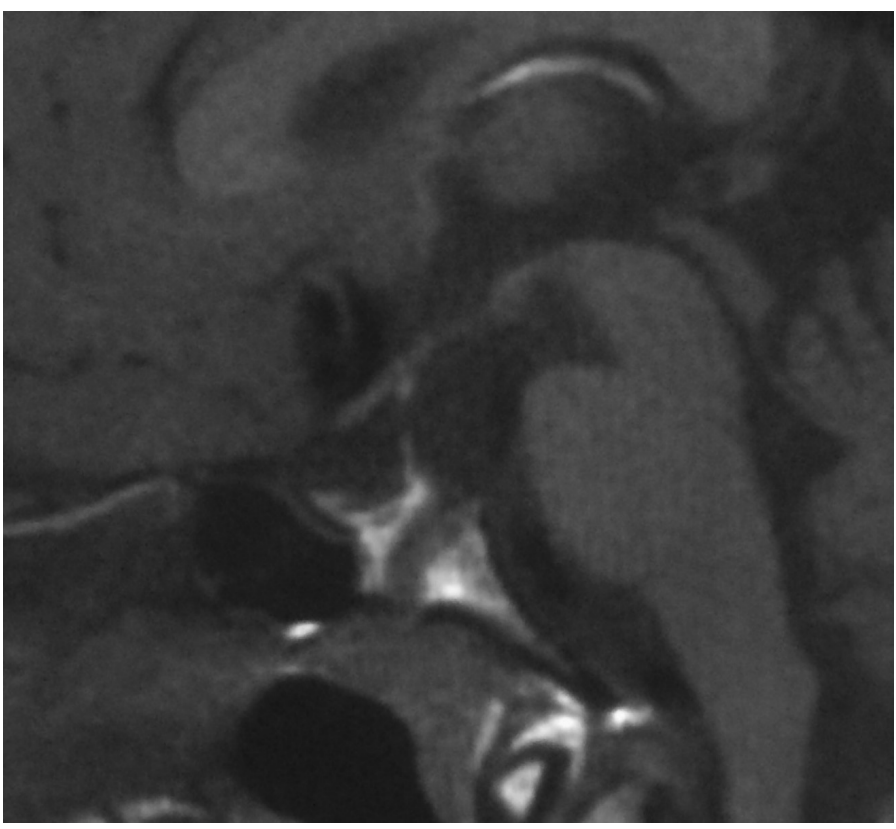

Figure 4. Magnetic resonance imaging (MRI) image from the patient's mother. Her pituitary MRI showed a thin pituitary stalk and hypoplasia of the adenohypophysis

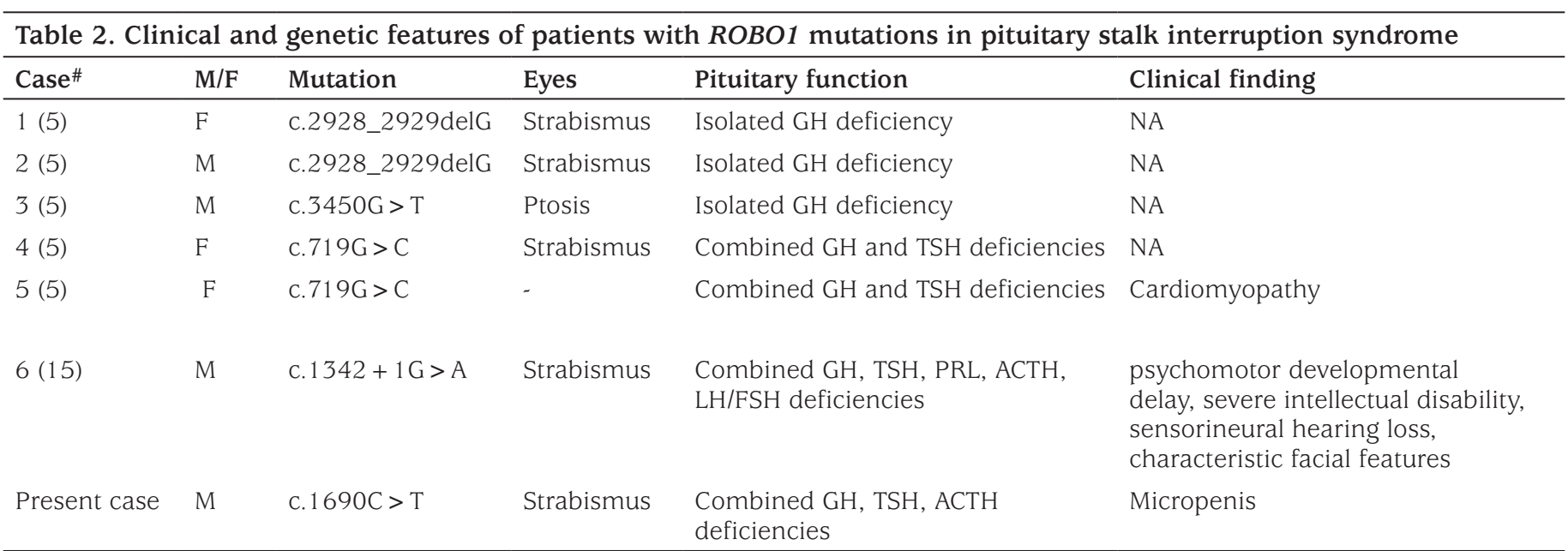

GH: growth hormone, TSH: thyrotropin, PRL: prolactin, ACTH: adrenocorticotropic hormone, LH: luteinizing hormone, FSH: follicle-stimulating hormone, NA: not avalible, M: male, F: female

\#Ref. 5, 15 
Dateki et al (15) identified a novel homozygous splice site mutation in $R O B O 1$ (c. $1342+1 \mathrm{G}>\mathrm{A}$ ) in a 5 year-old boy. The patient had CPHD, psychomotor developmental delay, severe intellectual disability, sensorineural hearing loss, strabismus, and characteristic facial features. Their findings suggest $R O B O 1$ gene as one of the potential causative genes of PSIS.The clinical phenotype of the patients harboring the ROBO1 mutation varied in terms of the ocular and endocrine manifestations. In our report, by using next generation sequencing (NGS) technology, we identified a maternal missense mutation (c.1690C > T, p.Pro564Ser) in the ROBO1 gene in a case diagnosed with PSIS and CPHD. This variant was predicted to be possibly pathogenic by Polyphen-2 and SIFT. Multiple amino acid sequence alignments showed that p.Pro 564 is highly conserved across various species including primates, other mammals birds and fish. All these findings suggest that this variant could play an important role in disease causation. Other patients who harbor $R O B O 1$ mutations have been reported to share some phenotypic features with the present patients. Four cases presented with strabismus and one case presented with ptosis. These data suggest that mutations in $R O B O 1$ contribute to ocular anomalies. Cardiomyopathy was seen in one patient and one patient had psychomotor developmental delay. More cases are needed to elucidate the relationship between genotype and phenotype. The ROBO1 and its ligand Slit are known to influence axon guidance and central nervous system patterning in both vertebrate and nonvertebrate systems (16). Missing expression of ROBO1 could lead to ectopic differentiation of forebrain neurons. The chemo repulsive ligand Slit and its receptors of the ROBO family are expressed in the developing and adult brain (17) and are crucially involved in the formation of midline commissures. Slit2 and Slit1/2 double knockout animals display defects in corticothalamic and thalamocortical targeting, callosal and hippocampal commissure projections (18) and defects in the formation of the optic chiasm. Calloni et al (19) described a 9-year-old boy with severe intellectual disability, absence of the transverse pontine fiber, thinning of the anterior commissure and corpus callosum, and compound heterozygous variants in the ROBO1 gene. These findings strongly suggest that human $R O B O 1$ variants could result in neurodevelopmental disorders. Our patient was subsequently found to exhibit a wide range of symptoms, including classic CPHD and right strabismus. Thus the ROBO1 gene may be one of the potential causative genes for PSIS and CPHD. Bjorke et al (20) showed that Slit signaling is necessary to inhibit the initiation of oculomotor neuron development. Oculomotor axons at the midline crossing are characterized by an axon-like process that forms from the cell body as a secondary axon. It may be possible that this repolarization is subject to $\mathrm{ROBO}$ regulation. Overall, the introduction of NGS technology in the diagnostic workflow will lead to the identification of novel genetic determinants in pediatric patients with pituitary defects $(21,22)$. There is mounting evidence that ROBO variants are associated with ocular as well as pituitary abnormalities.Additional examples of $R O B O 1$ variants and clinical PSIS cases are needed to explore the function of $R O B O 1$ and its effect on human embryogenesis and organogenesis.

\section{Ethics}

Informed Consent: Consent form was filled out by all participants.

Peer-review: Externally and internally peer-reviewed.

\section{Authorship Contributions}

Concept: Ziqin Liu, Design: Xiaobo Chen, Data Collection or Processing: Ziqin Liu, Analysis or Interpretation: Ziqin Liu, Literature Search: Xiaobo Chen, Writing: Ziqin Liu.

Financial Disclosure: The authors declare that this study has received no financial support.

\section{References}

1. El Chehadeh-Djebbar S, Callier P, Masurel-Paulet A, Bensignor C, Méjean N, Payet M, Ragon C, Durand C, Marle N, Mosca-Boidron AL, Huet F, Mugneret F, Faivre L, Thauvin-Robinet C. 17q21.31 microdeletion in a patient with pituitary stalk interruption syndrome. Eur J Med Genet 2011;54:369-373. Epub 2011 Mar 30

2. Arrigo T, Wasniewska M, De Luca F, Valenzise M, Lombardo F, Vivenza D, Vaccaro T, Coradi E, Biason-Lauber A. Congenital adenohypophysis aplasia: clinical features and analysis of the transcriptional factors for embryonic pituitary development. J Endocrinol Invest 2006;29:208213.

3. Fujisawa I, Asato R, Nishimura K, Togashi K, Itoh K, Nakano Y, Itoh H, Hashimoto N, Takeuchi J, Torizuka K. Anterior and posterior lobes of the pituitary gland: assessment by $1.5 \mathrm{~T}$ MR imaging. J Comput Assist Tomogr 1987;11:214-220.

4. Argyropoulou M, Pérignon F, Brauner R, Brunelle F. Magnetic resonance imaging in the diagnosis of growth hormone deficiency. J Pediatr 1992;120:886-891.

5. Bashamboo A, Bignon-Topalovic J, Moussi N, McElreavey K, Brauner R. Mutations in the human ROBO1 gene in pituitary stalk interruption syndrome. J Clin Endocrinol Metab 2017;102:2401-2406.

6. Adzhubei I, Jordan DM, Sunyaev SR. Predicting functional effect of human missense mutations using PolyPhen-2. Curr Protoc Hum Genet 2013

7. Gray VE, Kukurba KR, Kumar S. Performance of computational tools in evaluating the functional impact of laboratory-induced amino acid mutations. Bioinformatics 2012;28:2093-2096. Epub 2012 Jun 8

8. Choi Y, Sims GE, Murphy S, Miller JR, Chan AP. Predicting the functional effect of amino acid substitutions and indels. PLoS One 2012;7:e46688. Epub 2012 Oct 8

9. Richards S, Aziz N, Bale S, Bick D, Das S, Gastier-Foster J, Grody WW, Hegde M, Lyon E, Spector E, Voelkerding K, Rehm HL; ACMG 
Laboratory Quality Assurance Committee. Standards and guidelines for the interpretation of sequence variants: a joint consensus recommendation of the American College of Medical Genetics and Genomics and the Association for Molecular Pathology. Genet Med 2015;17:405-424. Epub 2015 Mar 5

10. Bashamboo A, Bignon-Topalovic J, Rouba H, McElreavey K, Brauner R. A Nonsense Mutation in the Hedgehog Receptor CDON Associated With Pituitary Stalk Interruption Syndrome. J Clin Endocrinol Metab 2016;101:12-15. Epub 2015 Nov 3

11. Melo ME, Marui S, Carvalho LR, Arnhold IJ, Leite CC, Mendonca BB, Knoepfelmacher M. Hormonal, pituitary magnetic resonance, LHX4 and HESX1 evaluation in patients with hypopituitarism and ectopic posterior pituitary lobe. Clin Endocrinol (Oxf) 2007;66:95-102.

12. Reynaud R, Albarel F, Saveanu A, Kaffel N, Castinetti F, Lecomte P, Brauner R, Simonin G, Gaudart J, Carmona E, Enjalbert A, Barlier A, Brue T. Pituitary stalk interruption syndrome in 83 patients: novel HESX1 mutation and severe hormonal prognosis in malformative forms. Eur J Endocrinol 2011;164:457-465. Epub 2011 Jan 26

13. Pinto G, Netchine I, Sobrier ML, Brunelle F, Souberbielle JC, Brauner R. Pituitary stalk interruption syndrome: a clinical-biological-genetic assessment of its pathogenesis. J Clin Endocrinol Metab 1997;82:34503454.

14. Davis SW, Potok MA, Brinkmeier ML, Carninci P, Lyons RH, MacDonald JW, Fleming MT, Mortensen AH, Egashira N, Ghosh D, Steel KP, Osamura RY, Hayashizaki Y, Camper SA. Genetics, gene expression and bioinformatics of the pituitary gland. Horm Res 2009;71:101-115. Epub 2009 Apr 29

15. Dateki S, Watanabe S, Mishima H, Shirakawa T, Morikawa M, Kinoshita E, Yoshiura KI, Moriuchi H. A homozygous splice site ROBO1 mutation in a patient with a novel syndrome with combined pituitary hormone deficiency. J Hum Genet 2019;64:341-346. Epub 2019 Jan 28
16. Kidd T, Russell C, Goodman CS, Tear G. Dosage-Sensitive and Complementary Functions of Roundabout and Commissureless, Control Axon Crossing of the CNS Midline. Neuron 1998;20:25-33.

17. Marillat V, Cases O, Nguyen-Ba-Charvet KT, Tessier-Lavigne M, Sotelo C, Chédotal A. Spatiotemporal expression patterns of slit and robo genes in the rat brain. J Comp Neurol 2002;442:130-155.

18. Bagri A, Marin O, Plump AS, Mak J, Pleasure SJ, Rubenstein JL, TessierLavigne M. Slit proteins prevent midline crossing and determine the dorsoventral position of major axonal pathways in the mammalian forebrain. Neuron 2002;33:233-248.

19. Calloni SF, Cohen JS, Meoded A, Juusola J, Triulzi FM, Huisman TAGM, Poretti A, Fatemi A. Compound Heterozygous Variants in ROBO1 Cause a Neurodevelopmental Disorder With Absence of Transverse Pontine Fibers and Thinning of the Anterior Commissure and Corpus Callosum. Pediatr Neurol 2017;70:70-74. Epub 2017 Feb 2

20. Bjorke B, Shoja-Taheri F, Kim M, Robinson GE, Fontelonga T, Kim KT, Song MR, Mastick GS. Contralateral migration of oculomotor neurons is regulated by Slit/Robo signaling. Neural Dev 2016;11:18.

21. Karaca E, Buyukkaya R, Pehlivan D, Charng WL, Yaykasli KO, Bayram Y, Gambin T, Withers M, Atik MM, Arslanoglu I, Bolu S, Erdin S, Buyukkaya A, Yaykasli E, Jhangiani SN, Muzny DM, Gibbs RA, Lupski JR. WholeExome Sequencing Identifies Homozygous GPR161 Mutation in a Family with Pituitary Stalk Interruption Syndrome. J Clin Endocrinol Metab 2015;100:140-147.

22. Guo QH, Wang CZ, Wu ZQ, Qin Y, Han BY, Wang AP, Wang BA, Dou JT, Wu $\mathrm{XS}, \mathrm{Mu} Y \mathrm{YM}$. Multi-genic pattern found in rare type of hypopituitarism: a whole-exome sequencing study of Han Chinese with pituitary stalk interruption syndrome. J Cell Mol Med 2017;21:3626-3632. Epub 2017 Jul 14 